\title{
SPATIAL DECAY ESTIMATES IN TRANSIENT HEAT CONDUCTION*
}

\author{
BY \\ C. O. HORGAN (Michigan State University) \\ L. E. PAYNE (Cornell University) \\ L. T. WHEELER (University of Houston)
}

\begin{abstract}
The spatial decay of solutions to initial-boundary value problems for the heat equation in a three-dimensional cylinder, subject to non-zero boundary conditions only on the ends, is investigated. It is shown that the spatial decay of end effects in the transient problem is faster than that for the steady-state case. Qualitative methods involving second-order partial differential inequalities for quadratic functionals are first employed. The explicit spatial decay estimates are then obtained by using comparison principle arguments involving solutions of the one-dimensional heat equation. The results give rise to versions of Saint-Venant's principle in transient heat conduction.
\end{abstract}

1. Introduction. The spatial decay of solutions of parabolic differential equations has been the subject of much recent attention [1-5]. These studies were motivated by a desire to establish, for parabolic equations, decay estimates analogous to those obtained for elliptic equations in the investigation of Saint-Venant's principle in elasticity theory. (See [6] for a review of recent work on Saint-Venant's principle.)

Boley [7-10] was apparently the first to raise the issue of the validity of a Saint-Venant principle for transient heat conduction. In $[8,10]$ versions of Saint-Venant's principle for non-cylindrical domains, similar to those of von Mises and Sternberg for elasticity, were considered. Using explicit upper bounds for solutions of half-space problems obtained from appropriate fundamental solutions, Boley [10] observed that the spatial influence of transient effects was even more localized than that of the steady-state. The validity of a Saint-Venant principle of the more traditional type, for cylindrical domains subject to non-zero boundary conditions on the ends only, was considered in [7, 9]. An illustrative example involving the explicit solution of a specific initial-boundary value problem for a semi-infinite rectangular strip is discussed in [9], pp. 186-187. In this example, it is seen that the spatial decay of end effects at any time $t$ in the transient problem is faster than that for the steady-state case [9].

${ }^{*}$ Received by the editors April 27, 1983. The work of C. O. Horgan was supported by NSF Grant MEA-78-26071 while that of L. E. Payne was supported by NSF Grant MCS-79-19358. 
The work described in [1-4] and in the present paper is concerned with initial-boundary value problems for parabolic equations on cylindrical-type domains subject to non-zero boundary conditions only on the ends. Qualitative methods are used to establish the exponential decay of solutions of such problems with distance from the ends and estimates for the decay rates are obtained. For classical linear heat conduction, it was shown by Knowles in [3] (see also Eqs. (2.6), (2.7) below) that end effects for the transient case decay spatially at least as rapidly as do their counterparts in the steady-state case. This result was established in [3] using arguments based on differential inequalities for quadratic functionals. A similar result can be obtained on employing the maximum principle [4]. Our purpose in the present paper is to employ qualitative methods to establish stronger results, analogous to those of $[7,9]$, namely that the spatial decay of end effects at time $t$ in the transient problem is faster than that for the steady-state case. For simplicity of presentation, we confine attention to the initial boundary-value problem of classical linear heat conduction in a three-dimensional semi-infinite cylinder.

2. Statement of problem. Let $R$ denote the interior of a semi-infinite cylinder, whose cross-section is bounded by a piecewise smooth simple closed curve. Choose cartesian coordinates $x_{1}, x_{2}, x_{3}$ with the origin at one end of the cylinder and the $x_{3}$-axis parallel to the generators. Let $S_{z}$ denote the open cross-section of $R$ for which $x_{3}=z, z \geqslant 0$, with corresponding curvilinear boundary denoted by $\partial S_{z}$. Suppose that $R$ is occupied by a homogeneous isotropic heat conductor of constant thermal diffusivity and that the lateral surface $L$ of $R$ is maintained at zero temperature. If $u(\mathbf{x}, t)=u\left(x_{1}, x_{2}, x_{3}, t\right)$ is the time-dependent temperature field ${ }^{\prime}$ in $R$, then, using appropriate dimensionless variables $\mathbf{x}$ and $t$, the dimensionless temperature $u$ must satisfy the heat equation ${ }^{2}$

$$
u,_{i i}-\dot{u}=0 \text { in } R \times(0, \infty),
$$

as well as the boundary condition

$$
u=0 \quad \text { on } L \times[0, \infty)
$$

We consider only temperature fields which are initially uniform and hence impose the initial condition

$$
u(\mathbf{x}, 0)=0, \quad \mathbf{x} \in R .
$$

To establish a principle of Saint-Venant type for transient heat conduction, it is sufficient to consider the end condition

$$
u=f \text { on } \overline{S_{0}} \times[0, \infty)
$$

\footnotetext{
'For simplicity the function $u$ is assumed to be a classical solution in $R \times[0, \infty)$ which takes on its boundary and initial values in the strict sense. It will be clear, however, that the results of this paper will hold for appropriately formulated weak solutions.

${ }^{2}$ The superposed dot denotes time differentiation, with the usual indicial notation being used for spatial derivatives.
} 
where $f\left(x_{1}, x_{2}, t\right)$ is a prescribed function which is such that

$$
f\left(x_{1}, x_{2}, 0\right)=0 \text { on } \overline{S_{0}} \text {. }
$$

It is assumed that $f$ is such that a solution $u$ (with required smoothness) of the initial-boundary value problem (2.1)-(2.4) exists.

Since the boundary conditions for $u$ are non-zero only on the end $x_{3}=0$, we anticipate that, provided $u(\mathbf{x}, t)$ is appropriately restricted as $x_{3} \rightarrow \infty$, it should not differ appreciably from the trivial (zero) temperature field except near the end $x_{3}=0$. Indeed, it has been shown by Knowles [3] that if $u \rightarrow 0$ (uniformly in $x_{\alpha}, t$ ) as $x_{3} \rightarrow \infty$, then at each time instant, $u(\mathbf{x}, t)$ decays spatially in $x_{3}$ at an exponential rate. The main result of [3] for the problem (2.1)-(2.4) may be described as follows: Let

$$
E(z, t)=\int_{0}^{t} \int_{R_{z}} u,{ }_{i} u,{ }_{i} d V d \tau+\frac{1}{2} \int_{R_{z}} u^{2} d V, \quad z \geqslant 0, t \geqslant 0,
$$

where $u$ satisfies (2.1)-(2.4). Here $R_{z}$ denotes that portion of the cylinder $R$ in which $z<x_{3}$. Then it is shown in [3] (see also [6]) that if $u \rightarrow 0$ (uniformly in $x_{\alpha}, t$ ) as $x_{3} \rightarrow \infty$, then

$$
E(z, t) \leqslant E(0, t) e^{-2\left(\lambda_{1}\right)^{1 / 2} z}, \quad z \geqslant 0, t \geqslant 0,
$$

where $\lambda_{1}$ is the first (positive) eigenvalue of the (fixed membrane) problem

$$
\begin{gathered}
\phi,_{\alpha \alpha}+\lambda \phi=0 \text { on } S_{z}, \\
\phi=0 \text { on } \partial S_{z} .
\end{gathered}
$$

The estimated decay rate $2\left(\lambda_{1}\right)^{1 / 2}$ in $(2.7)$ is identical with the (best possible) decay rate for the energy associated with the steady-state $(u=u(\mathbf{x}))$ analog of $(2.1),(2.2),(2.4)$ (see e.g. [6]). Thus end effects for transient heat conduction decay spatially at least as rapidly as do their counterparts in the steady case. As was observed in [3], this result might be expected since some solutions of (2.1) tend to solutions of Laplace's equation as $t \rightarrow \infty$.

The energy decay estimate (2.7) for the functional (2.6) was established in [3] using a technique involving first order ordinary differential inequalities, in which only derivatives with respect to the spatial variable $x_{3}$ appear. Such methods have been widely used to establish spatial decay estimates for elliptic boundary-value problems (see [6]).

We note here that the energy decay estimate (2.7) may be used to obtain pointwise decay estimates (cf. [1]) for $u(x, t)$, with an estimated decay rate $\left(\lambda_{1}\right)^{1 / 2}$.

Our purpose in this paper is to establish that the solution $u(\mathbf{x}, t)$ of $(2.1)-(2.4)$ decays spatially at a rate which is, in general, faster than the exponential rate predicted by (2.7). Our main result (see Eq. (5.10) below) may be viewed as a generalization of that of Boley [7, 9], developed for a specific two-dimensional problem. As in [3], we use differential inequality techniques for quadratic functionals and first derive a differential inequality which has spatial derivatives only with respect to $x_{3}$. In contrast with [3] however, this differential inequality is a second-order partial differential inequality, which may be reduced to a differential inequality for the one-dimensional heat operator. The final estimates are obtained using comparison principle arguments involving solutions of the one-dimensional heat equation. 
3. Derivation of differential inequality. For $u(\mathbf{x}, t)$ satisfying (2.1)-(2.4), we define the (non-negative) functional

$$
P(z, t)=[J(z, t)]^{1 / 2} \equiv\left(\int_{S_{z}} u^{2} d A\right)^{1 / 2}, \quad z \geqslant 0, t \geqslant 0 .
$$

In this section, we establish that $P(z, t)$ satisfies the differential inequality

$$
P_{z z} \geqslant \lambda_{1} P+\dot{P}, \quad z>0, t>0,
$$

where the subscript notation denotes differentiation with respect to the spatial variable $z$.

The derivation of (3.2) proceeds as follows: From its definition in (3.1), it follows that

$$
\begin{gathered}
J_{z}=\int_{S_{z}} 2 u u,{ }_{3} d A, \quad \dot{J}=\int_{S_{z}} 2 u \dot{u} d A, \\
J_{z z}=2 \int_{S_{z}}\left(u_{,_{3}}^{2}+u u,_{33}\right) d A .
\end{gathered}
$$

On using the differential equation (2.1), the divergence theorem and the boundary condition (2.2), we may write (3.4) as

$$
J_{z z}=\dot{J}+2 \int_{S_{z}} u_{, 3}^{2} d A+2 \int_{S_{z}} u,{ }_{\alpha} u,{ }_{\alpha} d A .
$$

Since, by (2.2), $u$ vanishes on the boundary $\partial S_{z}$ of $S_{z}, z \geqslant 0$, we have the inequality

$$
\int_{S_{z}} u,{ }_{\alpha} u,{ }_{\alpha} d A \geqslant \lambda_{1} \int_{S_{z}} u^{2} d A,
$$

where $\lambda_{1}$ is the first positive eigenvalue of (2.8), (2.9). Thus, on using (3.6) in (3.5), we obtain

$$
J_{z z} \geqslant 2 \lambda_{1} J+j+2 \int_{S_{z}} u_{,}^{2} d A .
$$

By virtue of (3.3) and the fact that $J \geqslant 0$, this may be written as

$$
J J_{z z}-\left(J_{z}\right)^{2} / 2 \geqslant 2\left[\left(\int_{S_{z}} u^{2} d A\right)\left(\int_{S_{z}} u_{{ }_{3}}{ }_{3} d A\right)-\left(\int_{S_{z}} u u,{ }_{3} d A\right)^{2}\right]+2 \lambda_{1} J^{2}+J \dot{J},
$$

and so, by Schwarz's inequality, we have

$$
J J_{z z}-\left(J_{z}\right)^{2} / 2 \geqslant 2 \lambda_{1} J^{2}+J j .
$$

On recalling the definition of $P(z, t)$ in (3.1), it may be readily verified that the inequalities (3.9) and (3.2) are equivalent, and so the derivation of (3.2) is complete. Note that no assumption has yet been made on the behavior of $u$ as $x_{3} \rightarrow \infty$.

4. A comparison result for solutions of (3.2). By virtue of its definition in (3.1), and the initial and boundary conditions (2.3), (2.4) for $u$, the function $P(z, t)$ satisfying the differential inequality (3.2), is subject to the initial and boundary conditions

$$
P(z, 0)=0, \quad z \geqslant 0,
$$




$$
P(0, t)=\left(\int_{S_{0}} f^{2} d A\right)^{1 / 2} \equiv g(t) \geqslant 0, \quad t \geqslant 0 .
$$

We assume further the following asymptotic behavior for $P(z, t)$ :

$$
P(z, t) \rightarrow 0 \quad \text { (uniformly in } t \text { ) as } z \rightarrow \infty .
$$

It will be clear a posteriori that such a strict decay hypothesis (4.3) can be relaxed. We observe that, in view of (2.5), the prescribed function $g(t)$ in (4.2) is such that

$$
g(0)=0 \text {. }
$$

In this section, we show that $P(z, t)$ can be bounded above by the solution to a related initial-boundary value problem for the one-dimensional heat equation.

Let

$$
P(z, t)=e^{-\lambda_{1} t} v(z, t) .
$$

Then, it follows from (3.2), (4.1)-(4.3), that $v(z, t)$ must satisfy

$$
\begin{gathered}
\mathcal{L} v \equiv v_{z z}-\dot{v} \geqslant 0, \quad z>0, t>0, \\
v(z, 0)=0, \quad z \geqslant 0, \\
v(0, t)=e^{\lambda_{1} t} g(t) \geqslant 0, \quad t \geqslant 0, \\
v(z, t) \rightarrow 0 \text { (uniformly in } t \text { ) as } z \rightarrow \infty .
\end{gathered}
$$

In view of its definition in (4.5), $v(z, t) \geqslant 0$ for $z \geqslant 0, t \geqslant 0$. We note that this result could also be deduced directly from (4.6)-(4.9) on using the maximum principle for parabolic differential inequalities [11].

An upper bound for $v(z, t)$ can now be obtained in terms of the solution of an initial-boundary value problem for the one-dimensional heat equation. Let $w(z, t)$ be such that

$$
\begin{gathered}
\mathcal{L} w=0, \quad z>0, t>0, \\
w(z, 0)=v(z, 0)=0, \quad z \geqslant 0, \\
w(0, t)=v(0, t)=e^{\lambda_{1} t} g(t), \quad t \geqslant 0, \\
w(z, t) \rightarrow 0 \text { (uniformly in } t \text { ) as } z \rightarrow \infty .
\end{gathered}
$$

Then, it follows immediately from the maximum principle for the heat equation (see e.g. [11], Chapter 3) that

$$
v \leqslant w, \quad z \geqslant 0, \quad t \geqslant 0,
$$

and so, on using (4.5), we have established the upper bound

$$
P(z, t) \leqslant e^{-\lambda_{1} t} w(z, t) .
$$

In the next section, we shall write out an explicit representation for the (unique) solution $w(z, t)$ of the problem (4.10)-(4.13) and thus obtain, from (4.15), an explicit upper bound for the functional $P(z, t)$. 
5. Spatial decay estimates. The solution to the initial-boundary value problem (4.10)(4.13) for the one-dimensional heat equation is, of course, well-known and can be found in many standard textbooks. Thus, for example, from [12], p. 208, we have

$$
w(z, t)=\frac{1}{2(\pi)^{1 / 2}} \int_{0}^{t} z(t-\tau)^{-3 / 2} e^{-z^{2} / 4(t-\tau)} e^{\lambda_{1} \tau} g(\tau) d \tau,
$$

which we rewrite in the form

$$
w(z, t)=\frac{1}{2(\pi)^{1 / 2}} e^{\lambda_{1} t} \int_{0}^{t} z \tau^{-3 / 2} e^{-\left(z^{2} / 4 \tau+\lambda_{1} \tau\right)} g(t-\tau) d \tau .
$$

Hence, on using (4.15), we obtain the upper bound

$$
P(z, t) \leqslant \frac{1}{2(\pi)^{1 / 2}} \int_{0}^{t} z \tau^{-3 / 2} e^{-\left(z^{2} / 4 \tau+\lambda_{1} \tau\right)} g(t-\tau) d \tau,
$$

and so

$$
P(z, t) \leqslant\left(\max _{[0, t]} g(s)\right) G(z, t),
$$

where $G(z, t)$ is defined by

$$
G(z, t)=\frac{1}{2(\pi)^{1 / 2}} \int_{0}^{t} z \tau^{-3 / 2} e^{-\left(z^{2} / 4 \tau+\lambda_{1} \tau\right)} d \tau .
$$

Thus, in view of (3.1), (4.2), our main estimate (5.4) for the solution $u$ of the original problem (2.1)-(2.4), subject to the hypothesis (4.3), reads

$$
\left(\int_{S_{z}} u^{2} d A\right)^{1 / 2} \leqslant\left[\max _{[0, t]}\left(\int_{S_{0}} f^{2} d A\right)^{1 / 2}\right] G(z, t), \quad z \geqslant 0, t \geqslant 0,
$$

with $G(z, t)$ given in (5.5).

The result (5.6) provides a mean-square estimate on the cross-section $S_{z}$ for the solution of (2.1)-(2.4). It is possible to obtain from (5.6) a pointwise estimate for $u$, uniformly valid on $R \times[0, \infty)$ (cf. [6]) but we shall not pursue this here. Our main concern in the remainder of the paper is to assess the spatial decay character of the estimate (5.6), with emphasis on the contrast between the steady-state and transient cases.

For our purpose, it is convenient to express $G(z, t)$ in a different form from that of (5.5). Using the change of variables $s^{2}=z^{2} / 4 \tau$, it is easily shown from (5.5) that

$$
G(z, t)=2(\pi)^{-1 / 2} \int_{z / 2 t^{1 / 2}}^{\infty} e^{-\left(s^{2}+\lambda_{1} z^{2} / 4 s^{2}\right)} d s .
$$

The desired final expression for $G(z, t)$ now follows on employing a representation for the definite integral in (5.7) given by Abramowitz and Stegun [13], p. 304. Thus, it can be readily shown from (5.7) that $^{3}$

$$
G(z, t)=\frac{1}{2}\left[e^{-\left(\lambda_{1}\right)^{1 / 2} z} \operatorname{erfc}\left\{\frac{z}{2 t^{1 / 2}}-\left(\lambda_{1} t\right)^{1 / 2}\right\}+e^{\left(\lambda_{1}\right)^{1 / 2} z} \operatorname{erfc}\left\{\frac{z}{2 t^{1 / 2}}+\left(\lambda_{1} t\right)^{1 / 2}\right\}\right],
$$

\footnotetext{
${ }^{3}$ See also Carslaw and Jaeger [14], p. 134.
} 
where the complementary error function $\operatorname{erfc}(x)$ is defined by

$$
\operatorname{erfc}(x)=2(\pi)^{-1 / 2} \int_{x}^{\infty} e^{-\zeta^{2}} d \zeta .
$$

Thus, our main result (5.6) may now be written in the form

$$
\left(\int_{S_{z}} u^{2} d A\right)^{1 / 2} \leqslant\left[\max _{[0, t]}\left(\int_{S_{0}} f^{2} d A\right)^{1 / 2}\right] G(z, t), \quad z \geqslant 0, t \geqslant 0,
$$

where $G(z, t)$ is given by $(5.8)$.

From (5.5), it follows that $G$ is monotonically increasing in $t$. Therefore, since

$$
\lim _{x \rightarrow-\infty} \operatorname{erfc}(x)=2 \operatorname{erfc}(0)=2, \quad \lim _{x \rightarrow+\infty} \operatorname{erfc}(x)=0,
$$

(5.8) furnishes

$$
G(z, t) \leqslant \lim _{\tau \rightarrow \infty} G(z, \tau)=e^{-\left(\lambda_{1}\right)^{1 / 2} z}
$$

Thus, from (5.10), it follows that

$$
\left(\int_{s_{z}} u^{2} d A\right)^{1 / 2} \leqslant \max _{[0, t]}\left(\int_{S_{0}} f^{2} d A\right)^{1 / 2} e^{-\left(\lambda_{1}\right)^{1 / 2} z}, \quad z \geqslant 0, t \geqslant 0 .
$$

This bound, yielding an estimated decay rate appropriate for a steady-state, is analogous to the result (2.7) of Knowles [3].

The estimate (5.10) provides a sharper result in the presence of transient behavior. In Fig. 1, we introduce the parameter $\tau=\left(\lambda_{1} t\right)^{1 / 2}$ and plot curves of $G(z, t)$ versus $\left(\lambda_{1}\right)^{1 / 2} z$ for $\tau$ ranging from 0.25 to $\infty$, which corresponds to the steady-state. It is seen that the decay of end effects in the transient problem is indeed faster than that for the steady case, reinforcing the observations made by Boley [7,9]. (The solution of the specific problem considered on p. 187 of [9] has a form similar to the expression for $G(z, t)$ given in (5.8), with curves corresponding to those of Fig. 1 also given on p. 186 of [9].)

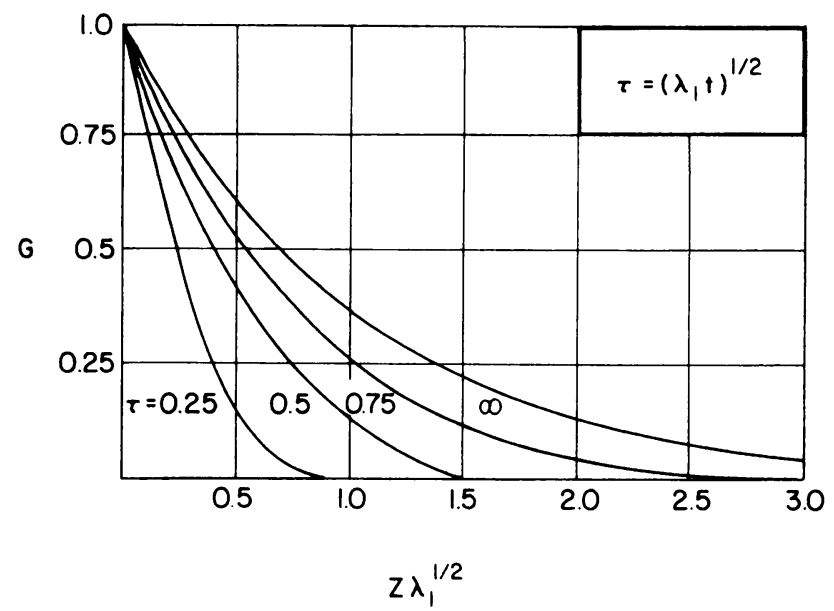

Fig. 1. The function $G(z, t)$ of Eqs. (5.5), (5.8). When $\tau=\infty$ (steady-state), $G=e^{-\left(\lambda_{1}\right)^{1 / 2} z}$. 
We note that estimates simpler than (5.10) may be obtained on using various upper bounds for $G(z, t)$ as given in (5.8). Thus, on integration by parts in (5.9), it can be shown that (cf. [13], p. 298)

$$
\sqrt{\pi} \operatorname{erfc}(x)<e^{-x^{2}} / x \text { for } x>0
$$

Accordingly, for $z>2\left(\lambda_{1}\right)^{1 / 2} t,(5.8),(5.10)$ yield the estimate

$$
\left(\int_{S_{z}} u^{2} d A\right)^{1 / 2} \leqslant\left[\max _{[0, t]}\left(\int_{S_{0}} f^{2} d A\right)^{1 / 2}\right] \frac{2 z(t / \pi)^{1 / 2} e^{-\lambda_{1} t}}{z^{2}-4 \lambda_{1} t^{2}} e^{-z^{2} / 4 t},
$$

which shows explicitly that, for fixed $t$, the spatial decay is ultimately controlled by the factor $\exp \left(-z^{2} / 4 t\right)$ rather than the factor $\exp \left[-\left(\lambda_{1}\right)^{1 / 2} z\right]$ found in the steady-state case.

A more delicate analysis would show that the decay estimate (5.15) remains valid if (4.3) is replaced by a weaker hypothesis such as: $e^{-k_{1} z}[P(z, t)+|(\partial / \partial z) P(z, t)|] \rightarrow 0$ uniformly in $t$ as $z \rightarrow \infty$ for an appropriately chosen $k_{1}$. In the interests of simplicity, however, we have imposed the stronger hypothesis (4.3).

We remark finally that from (5.3) it is possible to derive different estimates from that indicated by (5.4). For instance, instead of using the maximum value of $g(t-\tau)$, we could have employed weighted $L_{p}$ inequalities and obtain bounds for $\int_{S_{z}} u^{2} d A$ in terms of weighted $L_{p}$ norms of $f$.

6. Concluding remarks. An analysis similar to the foregoing may also be carried out for the problem analogous to (2.1)-(2.4) where the Dirichlet boundary conditions (2.2), (2.4) are replaced by boundary conditions of Neumann type. Such a problem was treated in [3] (see also [9]) and corresponds more closely to the traction boundary-value problem of elasticity where consideration of Saint-Venant's principle first arose (see e.g. [6]). Thus instead of (2.2), (2.4), one has the boundary conditions

$$
\begin{gathered}
\frac{\partial u}{\partial n}=0 \quad \text { on } L \times[0, \infty), \\
u,_{3}=h \quad \text { on } \bar{S}_{0} \times[0, \infty),
\end{gathered}
$$

where the prescribed function $h\left(x_{1}, x_{2}, t\right)$ is self-equilibrated:

$$
\int_{S_{0}} h d A=0, \quad t \geqslant 0
$$

For the Neumann problem, Knowles [3] obtains a result identical to (2.7) where $\lambda_{1}$ is replaced by $\mu_{1}$, the first positive eigenvalue of the (free membrane) problem

$$
\begin{gathered}
\phi_{, \alpha \alpha}+\mu \phi=0 \text { on } S_{z}, \\
\frac{\partial \phi}{\partial n}=0 \text { on } \partial S_{z} .
\end{gathered}
$$

In adapting the analysis of the present paper to the Neumann problem, it is straightforward to establish the differential inequality (3.2), with $\lambda_{1}$ replaced by $\mu_{1}$. A comparison result of the form (4.15) also follows (with $\lambda_{1}$ replaced by $\mu_{1}$ ) on invoking the strong form of the maximum principle [11]. The detailed calculations analogous to those of Sec. 5 are 
slightly modified but still can be shown to yield a result of the form (5.10), leading to similar conclusions to those reached for the Dirichlet problem.

In conclusion, we observe that a spatial decay estimate of the form (2.7), appropriate for the steady-state, has been obtained in [15] for solutions of the third-order pseudoparabolic equation

$$
u_{,_{i i}}+\eta \dot{u},_{i i}-\dot{u}=0 \text { on } R \times[0, \infty) .
$$

Here $\eta$ is a positive constant. (See also [16] for a generalization of the work of [5] to include such equations.) Equations of this type have been shown to model a wide variety of physical phenomena (see e.g. [17]), such as two temperature heat conduction theory. It remains to be seen whether the arguments of the present paper can be extended to cover this case.

\section{REFERENCES}

[1] W. S. Edelstein, A spatial decay estimate for the heat equation, J. Appl. Math. Phys. (ZAMP), 20, 900-905 (1969)

[2] V. G. Sigillito, On the spatial decay of solutions of parabolic equations, J. Appl. Math. Phys. (ZAMP), 21, 1078-1081 (1970)

[3] J. K. Knowles, On the spatial decay of solutions of the heat equation, J. Appl. Math. Phys. (ZAMP), 22, $1050-1056(1971)$

[4] C. O. Horgan and L. T. Wheeler, Spatial decay estimates for the heat equation via the maximum principle, J. Appl. Math. Phys. (ZAMP), 27, 371-376 (1976)

[5] O. A. Oleinik and G. A. Yosifian, An analogue of Saint-Venant's principle and the uniqueness of solutions of boundary value problems for parabolic equations in unbounded domains, Russian Math. Surveys, 31, 153-178 (1976)

[6] C. O. Horgan and J. K. Knowles, Recent developments concerning Saint-Venant's principle, Advances in Applied Mechanics (T. Y. Wu and J. W. Hutchinson, eds.), Vol. 23, Academic Press, New York (1983), 179-269

[7] B. A. Boley, The determination of temperature, stresses and deflections in two-dimensional thermoelastic problems, J. Aero. Sci., 23, 67-75 (1956)

[8] __ Some observations on Saint-Venant's principle, Proc. 3rd U.S. Nat. Cong. Appl. Mech., ASME, New York (1958), 259-264

[9] B. A. Boley and J. H. Weiner, Theory of thermal stresses, Wiley, New York, 1960

[10] B. A. Boley, Upper bounds and Saint-Venant's principle in transient heat conduction, Quart. Appl. Math. 18, 205-207 (1960)

[11] M. H. Protter and H. F. Weinberger, Maximum principles in differential equations, Prentice-Hall, New Jersey, 1967

12] A. N. Tikhonov and A. A. Samarski, Partial differential equations of mathematical physics, Holden-Day, New York, 1964

[13] M. Abramowitz and I. A. Stegun (eds.), Handbook of mathematical functions, Dover, New York, 1965

[14] H. S. Carslaw and J. C. Jaeger, Conduction of heat in solids, (2nd ed.), Oxford Univ. Press, Oxford, 1959

[15] C. O. Horgan and L. T. Wheeler, A spatial decay estimate for pseudoparabolic equations, Lett. Appl. Eng. Sci., 3, 237-243 (1975)

[16] G. I. Khil'kevich, An analogue of Saint-Venant's principle, the Cauchy problem and the first boundary-value problem in an unbounded domain for pseudoparabolic equations, Russian Math. Surveys, 36, 252-253 (1981)

[17] R. W. Carroll and R. E. Showalter, Singular and degenerate Cauchy problems, Academic Press, New York, 1976 